\title{
AUTOMATIC DOMINANT REGION SEGMENTATION FOR NATURAL IMAGES
}

\author{
A.Kalaivani ${ }^{1}$, S.P.Surender Nath ${ }^{2}$, Dr.S.Chitrakala ${ }^{3}$ \\ 1 Research Scholar, Easwari Engineering College, \\ kalaivanianbarasan@rediffmail.com \\ ${ }^{2}$ Anna University, Chennai, spsuren3egmail.com \\ ${ }^{3}$ Anna University, Chennai, chitraecs . annauniv.edu
}

\begin{abstract}
Image Segmentation segments an image into different homogenous regions. An efficient semantic based image retrieval system divides the image into different regions separated by color or texture sometimes even both. Features are extracted from the segmented regions and are annotated automatically. Relevant images are retrieved from the database based on the keywords of the segmented region In this paper, automatic image segmentation is proposed to obtained dominant region of the input natural images. Dominant region are segmented and results are obtained. Results are also recorded in comparison to JSEG algorithm.
\end{abstract}

\section{KEYWORDS}

Color Image Segmentation, Dominant Region, Edge Detection

\section{INTRODUCTION}

Machine vision system are composed of two sub-system which are classified as low-level vision and high-level vision systems. Low level vision systems perform image processing operation on the input image to produce output image of reduced noise or with certain enhanced features. Object recognition or scene interpretation can be done at the high level vision systems. Segmentation acts as a bridge between these two subsystems.

Image Segmentation is a most important step in image analysis and pattern recognition. Image Segmentation is a most difficult task in image processing. Image segmentation is the process of dividing the images into different regions based on specific criteria which may be color , texture or even both. According to [1], " the image segmentation problem is basically one of psychophysical perception and not susceptible to a purely analytical solution".

A formal definition of image segmentation is as follows [2]: If $\mathrm{P}()$ is a homogencity predicate defined on groups of connected pixels, then segmentation is a partition of the set $\mathrm{F}$ into connected subsets or regions $\left(\mathrm{S}_{1}, \mathrm{~S}_{2}, \ldots . \mathrm{S}_{3}\right)$ such that

n

$\mathrm{U} \mathrm{S}_{\mathrm{i}}=\mathrm{F}$ with $\mathrm{S}_{\mathrm{i}} \cap \mathrm{Sj}=\phi(\mathrm{i}<>\mathrm{j})$

$\mathrm{i}=1$

David C. Wyld (Eds) : ICCSEA, SPPR, CSIA, WimoA - 2013

pp. 273-280, 2013. () CS \& IT-CSCP 2013

DOI : 10.5121/csit.2013.3528 
The uniform predicate $\mathrm{P}\left(\mathrm{S}_{\mathrm{i}}\right)=$ true for all regions, $\mathrm{Si}$ and $\mathrm{P}\left(\mathrm{S}_{\mathrm{i}} U \mathrm{~S}_{\mathrm{j}}\right)=$ false when $\mathrm{i}<>\mathrm{j}$ and $\mathrm{S}_{\mathrm{i}}$ and $\mathrm{S}_{\mathrm{i}}$ are neighbors.

Color Image Segmentation mainly depends on two properties of the intensity values. The former property is discontinuity where the images are partitioned on sharp changes in intensity. The latter property is similarity in which the image is partitioned based upon a predefined similarity criteria. Taking into account of these properties several image segmentation techniques have been proposed. Choice of the techniques mainly depends on the problem consideration.

\subsection{Color Image Segmentation Applications}

Color Image segmentation partition the given input color image into homogenous regions. Image segmentation is the first important step in image analysis. Image analysis includes wide applications in video surveillance, image retrieval, medical imaging analysis and object classification. Color image segmentation finds a place in many multimedia application such as indexing and management of data and also in the dissemination of information in the network.

\subsection{Color Image Segmentation Approaches}

Color Image segmentation divides the image pixel based on image features. While using color images, image pixels are represented by various number of color spaces such as RGB, XYZ or LUV. Color image segmentation attracted researchers, due to providing more information on color images available in a larges repository in web and also the speed of processing color images through PCs. Color Image Segmentation techniques can be classified as edge based segmentation, thresholding based segmentation, region based segmentation, clustering based segmentation and graph based segmentation.

Edge based segmentation specify segmentation based on the edges of the image. Thresholding is very simple which is used to separate objects from the background. The different types of thresholding algorithms are adaptive thresholding and otsu thresholding. Region based segmentation is the one in which regions of similar pixels are grouped together. Two efficient algorithms are seeded region growing and region split and merge methods.

A good segmenter should produce homogenous and uniform regions based on color or texture. The boundaries of each segment should be spatially accurate, smooth but not ragged. Adjacent regions should be different based on region characteristics.

In this paper, we consider the problem of color image segmentation of dominant region based on color for natural images. The rest of this paper is organized as follows. In Section 2, related work carried on color image segmentation are discussed. In Section 3, the proposed algorithm for automatic dominant region segmentation algorithm are presented. In Section 4, provides the experimental results and discussion. Conclusion are given in Section 6.

\section{LITERATURE REVIEW}

Siddhartha Bhattacharyya [9] discussed the classical methods of image segmentation ranging from filtering methods to statistical methods. Color Image segmentation done using latest technique such as neural networks, fuzzy logic, genetic algorithms and wavelet decomposition are also presented. Gajendra Singh Chandel[14], analyzed various segmentation algorithms such as 
edge-based segmentation, thresholding based segmentation and Region Based Segmentation Algorithms. Polak[8]. specify an evaluation metric for image segmentation of multiple objects. Nilima Kulkarni[13] presented color thresholding method for image segmentation of natural images. Muthukrishnan presented edge detection techniques for image segmentation. Felci Rajam[11] proposed SRBIR system which retrieves images based on semantic content by extracting the dominant foreground region in the image and learning the semantic concept with the help of SVM-BDT.

\section{PROPOSED AlgORITHM OVERVIEW}

Color Image Segmentation is used to identify region of interest and objects in the scene which are beneficial to image annotation or image analysis. For identifying the dominant region for natural images, natural color images of different object classes are taken as the input image. In any type of image the dominant region occupies most of the image foreground. So, segmentation of dominant region can easily identify the object.

Firstly, the input RGB color image is indexed and then converted into a gray scale image . Secondly, various image preprocessing techniques such as median filtering, range filtering are carried out to eliminate noise. The quality of the input image is enhanced by applying image sharpening technique. Thirdly, edges are detected from the preprocessed image by applying canny edge detection technique. Fourthly, by applying gray threshold method, a foreground object is separated from the background. Fifthly, dominant region identification are done to identify dominant region and finally segmented color image with dominant object highlights are produced.

Proposed Algorithm for extracting dominant region from a given input RGB image is as below

Step 1 : Input RGB image

Step 2 : Generate an Indexed Image of an input image

Step 3 : Convert RGB input image into a gray scale image

Step 4 : Apply Preprocessing technique

Step 5 : Detect edges of the object

Step 6 : Separate foreground object from background object

Step 7 : Identify and draw object boundaries

Step 8 : Display the segmented foreground object

Proposed automatic dominant region image segmentation for natural images is depicted in the flowchart and is given in figure 1. 


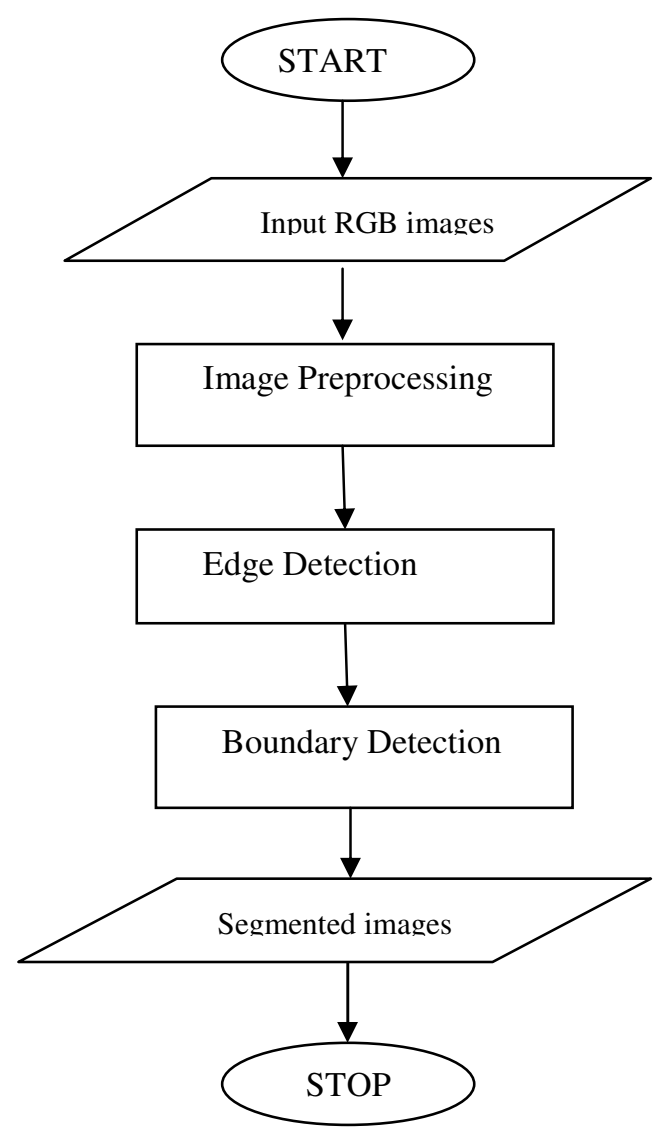

Fig . 1: Flowchart of the Proposed Algorithm

A given input RGB color image is converted into a gray scale image. The formula for converting RGB pixel to a gray pixel mapping the intensity value from 0 to 255 is given by equation 2 .

$$
\text { Intensity }=0.29289 * \text { red }+0.5870 * \text { green }+0.1140 * \text { blue }
$$

Image preprocessing is carried out to reduce image and improve image quality. The most popular median filtering is used for noise removal and preserve edges using $3 * 3$ neighborhood. Median filtered of an gray image is returned as output image for further image preprocessing steps. The function for performing median filtering is medfilt2() which is given by

$$
\mathrm{B}=\operatorname{medfilt} 2(\mathrm{~A})
$$

Various other preprocessing techniques are applied such as sharpening and range filtering on the median filtered image. Edges are detected for the preprocessed image .A large number of edge detecting technique are available in which canny edge detection is ideal since it returns the threshold value on which the regions are segmented. The function for performing canny edge detection is as below :

$$
\text { F=edge(I,'canny') }
$$


where $\mathrm{I}$ is the input gray scale image and $\mathrm{F}$ is the edge detected final image. Once the edges are detected, objects are identified by tracing the boundary. Final segmented objects are identified which is a black and white image. Color segmented image is obtained from the indexed image . Boundary is specified in white color to display the segmented image.
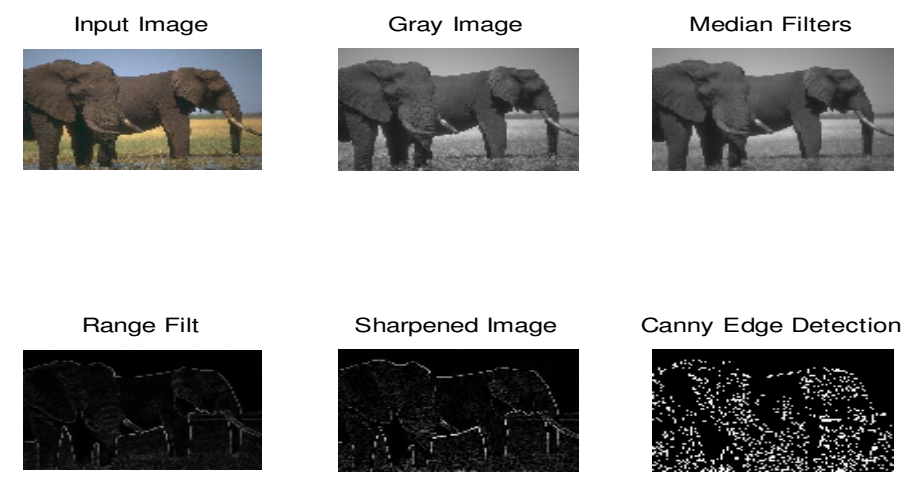

Figure 2: Stages of Image Segmentation- Image Preprocessing Stage
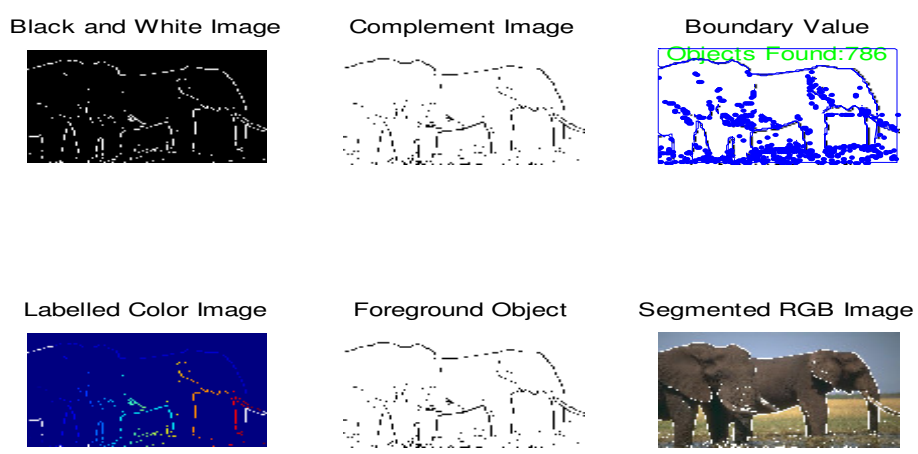

Figure 3: Stages of Image Segmentation- Edge Detection \& Gray Thresholding

\section{EXPERIMENTAL RESULTS AND DISCUSSION}

Berkeley Image Segmentation deals with benchmark datasets. Segmentation are done by the user with different number of segments. For the testing of the algorithm images are taken from these datasets and the segmented results are given in the table 1. 
Table 1. Segmented Results of Benchmark Datasets and Proposed Algorithm

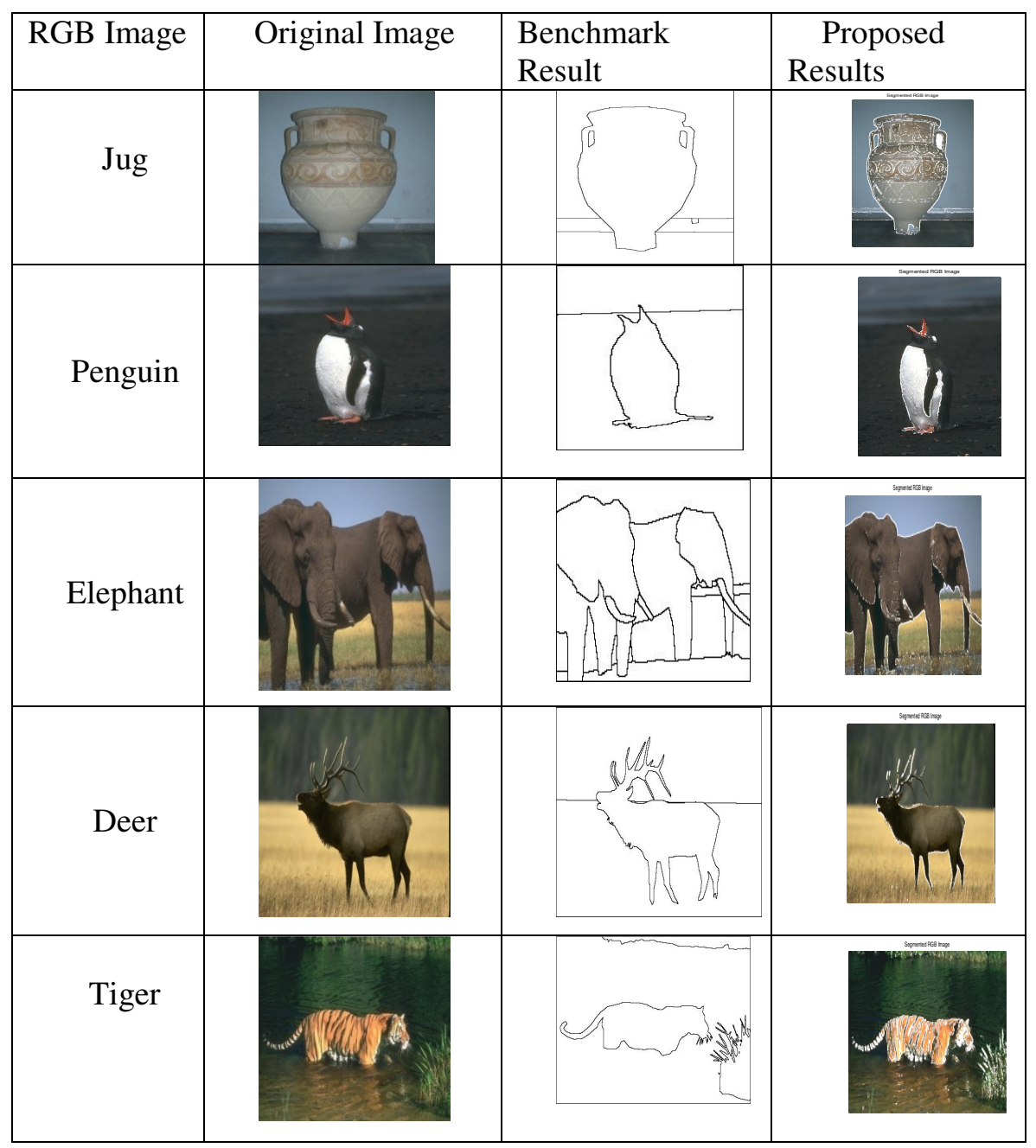

In region based Image retrieval system, most of the papers used JSEG image segmentation algorithm in which multi objects are segmented based on color and texture. But the algorithm deals with a lot of limitations such as shades due to the illumination, over segmentation. Visually, there is no clear boundary and is often over-segmented into several regions. Our proposed algorithm avoids the problem of over segmentation and a clear boundary is obtained for a dominating objects. 
Table 2. Segmented Results of JSEG and Proposed Algorithm

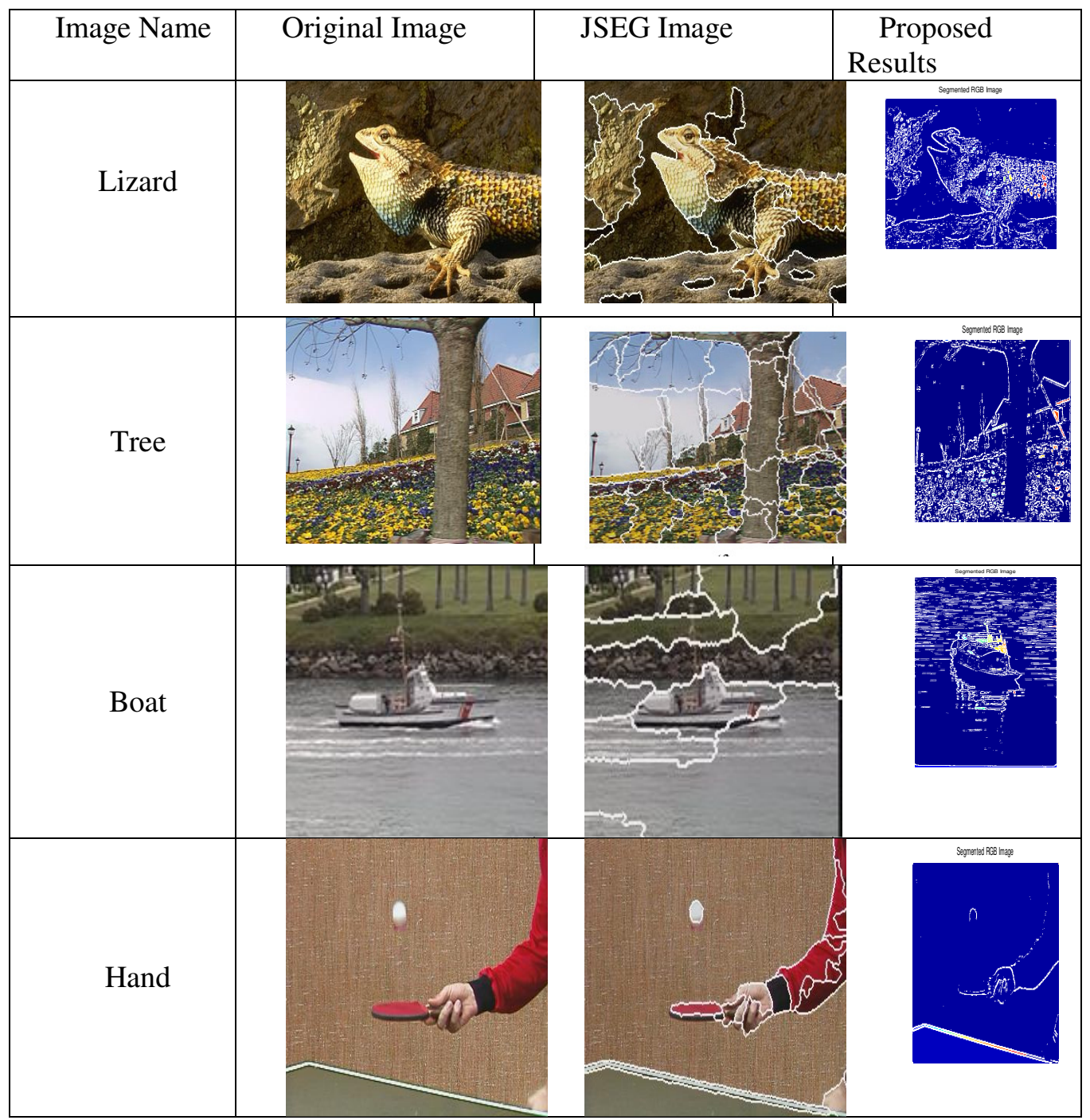

\section{CONCLUSIONS}

Color and texture are critical factors in human visual perception. Many segmentation approaches use both the factors to obtain homogenous regions for image segmentation. In this paper, a new approach for segmenting dominant region for natural images are proposed. Earlier algorithms such as Normalized Cut, JSEG, ICMAP algorithms all deal with a lot of computation and takes longer time to segment the image. Our Proposed algorithm is simple to implement and are able to get the desired segmented results for simple natural images concentrating on the dominant region. Our future work aims at classifying foreground region based on texture also. Regions can be separated and features can also be extracted for segmented regions. Further Image analysis can be done to come up with an efficient Image Retrieval System. 


\section{REFERENCES}

[1] K.S.Fu, J.K. Mui, : A survey on image segmentation, Pattern Recognition 13 (1981) 3-16.

[2] N.R.Pal, S.K. Pal : A review on Image segmentation techniques, Pattern Recognition 26 (9) (1993) 1277-1294.

[3] Lily Spirkovska, Ames Research Center, Moffett Field: A Summary of Image Segmentation Techniques, NASA, Ames Research Center (June1993) 1-11.

[4] H.D. Cheng, X.H.Jiang, Y.Sun, Jingli Wang: Color image segmentation : advances and prospects Pattern Recognition, Elsevier Science Limited (2001) 2259 - 2281.

[5] C.Carson, S.Belongie, H.Greenspan, J.Malik : Blobworld: Image segmentation using expectationmaximization and its application to image querying, IEEE Trans. Pattern Anal. Mach. Intell. , 8, (8) (2002) 1026-1038

[6] Frank Y.Shih,Shouxian Cheng, "Automatic seeded region growing for color imag segmentation",Image and Vision Computing 23(2005)877-886.

[7] Zhang,H.Fritts, J.E \& Goldman. S.A : Image Segmentation Evaluation: A survey unsupervised methods computer vision and image understanding, Computr vision and image understanding, 10, 2 (2008), 260-280.

[8] Polak, M.Zhang, H \& PI, M. : An evaluation metric for image segmentation of multiple objects , Image and Vision Computing, 27, 8 (2009), 1223-1227.

[9] Bhattacharyya: A Brief Survey on Image preprocessing and Segmentation Techniques , Journal of Pattern Recognition Research 1 (2011) 126-129.

[10] Muthukrishnan.R, M.Radha: Edge Detection Techniques for Image Segmentation, International Journal of Computer Science \& Information Technology, Vol.3, No.5 (2011), 259-267.

[11] Felci Rajam, S.Valli: SRBIR : Semantic Region Based Image Retrieval by Extracting the Dominant Region and Semantic Learning, Journal of Computer Science 7 , 3 (2011), 400-408.

[12] R.Muthukrishnan and M.Radha : Edge Detection Techniques for Image Segmentation, International Journal of Computer Science \& Information Technology, 3 ((2011) 259-267.

[13] Nilima Kulkarni: Color Thresholding Method for Image Segmentation of Natural Images , International Journal of Image, Graphics and Signal Processing, 1 (2012) 28-34.

[14] Gajendra Singh Chandel, Ravindra Kumar, Deepika khare, Sumita Verma : Analaysis of Image Segmentation Algorithms using Matlab, International Jounral of Engineering Innovation \& Research, Vol.1, Issue 1, (2012) 51-55. 